\title{
Licencias parentales y política social de la paternidad en España*
}

\author{
Lluís FLAQUER \\ Universitat Autònoma de Barcelona \\ Lluís.flaquer@uab.cat \\ Anna ESCOBEDO \\ Universitat de Barcelona \\ anna.escobedo@ub.edu
}

Recibido: 28-08-2013

Aceptado: 04-10-2013

\section{RESUMEN}

En el presente artículo analizamos en primer término el impacto diferencial de la paternidad y de la maternidad sobre las trayectorias laborales. El análisis longitudinal de datos de la Seguridad Social muestra como el nacimiento de un primogénito se configura como punto de inflexión a partir del cual en nuestro país divergen el conjunto de trayectorias laborales femeninas y masculinas de forma que el acceso a la paternidad aparece claramente asociado con una mayor dedicación al empleo por parte de los padres. Esta constatación nos induce a explorar el desarrollo de la política social de la paternidad en España en la medida en que la implicación paterna en el cuidado de los hijos constituye un factor clave tanto para lograr una mayor igualdad de oportunidades entre mujeres y hombres como para mejorar el nivel de bienestar infantil. Para ello, nos centramos en el desarrollo de las licencias parentales en relación a los hombres para pasar luego a estudiar desde una perspectiva comparada la evolución de la regulación del sistema de licencias parentales en nuestro país. A continuación analizamos datos y proponemos indicadores sobre la percepción de las prestaciones percibidas por padres tras el nacimiento de un hijo, a partir de la ampliación del permiso de paternidad en 2007, coincidiendo justo con el inicio de la crisis en nuestro país. Pese a los recortes y la crisis, nuestros datos arrojan como resultado que las tasas de uso de la licencia de paternidad sobre la población masculina potencialmente usuaria no ha dejado de aumentar entre 2008 y 2011, dándose sin embargo unas diferencias notables según comunidades autónomas, nivel educativo de los padres y distintas modalidades de empleo.

Palabras clave: nuevas paternidades, licencias parentales, permiso de paternidad, política social de la paternidad

\section{Parental leave and the social politics of fatherhood in Spain}

* La redacción de este artículo ha contado con la ayuda del proyecto "La implicación paterna en el cuidado de los hijos" (CSO2012-33476) financiado por el subprograma de Proyectos de Investigación Fundamental no Orientada en el marco del VI Plan Nacional de Investigación Científica, Desarrollo e Innovación Tecnológica 2008-2011. 


\begin{abstract}
In this article we analyse in the first place the different employment impact of fatherhood and motherhood. The analysis of longitudinal data from the Spanish Social Security shows how the birth of the first child is a turning point from which there appears to be a divergence between male and female employment trajectories so that for fathers this juncture is clearly associated with an intensification of their breadwinning role. This finding prompts us to explore the development of the social politics of fatherhood in Spain to the extent to which father involvement in the care of children constitutes a key factor to enhance equal opportunities between men and women as well as to improve child well-being levels. To this end, we focus on the emergence of parental leaves for men to turn then to study the evolving regulation of parental leave in Spain from a comparative perspective. In the final part of the article we analyse data and we propose indicators of paternity leave take-up, following the upgrading of this benefit in 2007, shortly before the start of the financial crisis. In spite of cutbacks resulting from the recession, our data indicate that paternity leave takeup rates in relation to potential male users have increased from 2008 to 2011, although significant variations appear by region, educational attainment and quality of employment.
\end{abstract}

Keywords: emerging new parenting styles, parental leave, paternity leave, social politics of fatherhood.

\title{
REFERENCIA NORMALIZADA
}

Flaquer, Ll, y Escobedo A., (2014). "Licencias parentales y política social de la paternidad en España". Cuadernos de Relaciones Laborales, Vol. 32, núm.1, p. 69-99.

SUMARIO: Introducción. 1. El impacto diferencial de la paternidad y la maternidad sobre el empleo. 2. Permisos y licencias de paternidad. 3. El sistema español de excedencias y prestaciones para el cuidado de hijos y familiares. 4. Análisis empírico de datos sobre el uso de licencias de paternidad y de maternidad percibidas por el padre. 4.1. Construcción de un indicador para estimar el uso de los permisos de paternidad y de maternidad disfrutados por padres. 4.2. Evolución y distribución territorial de las tasas de uso de las prestaciones de paternidad y maternidad percibidas por el padre. 4.3. Análisis de correlaciones sobre posibles factores que inciden en el uso de los permisos. 5. Conclusiones. 6. Bibliografía.

\section{Introducción}

En los últimos años las pesquisas sobre la política social de la paternidad han ido ganando terreno en la agenda de investigación social (Cabrera \& Peters, 2000; Hobson, 2002; O'Brien, 2004; Marsiglio \& Roy, 2012), fruto del interés creciente sobre la institucionalización de los modos de articulación entre familia, Estado de bienestar y mercado laboral y de la constatación de que la implicación paterna en el cuidado de los hijos es un factor clave tanto para lograr una mayor igualdad de oportunidades entre mujeres y hombres como para mejorar el nivel de bienestar infantil (Björnberg \& Kollind eds, 1996; Fusulier \& Marquet, 2007; Wilson \& Prior, 2011; Martial, 2012; Flaquer, 2013). En España existe una buena tradición de investigación sobre estas temáticas, que se remonta a años atrás y recientemente se advierte un renovado interés por la cuestión (Meil, 1997 y 2011; Valiente, 1997 y 2005; Alberdi y Escario, 2007; Lapuerta, Baizán \& González, 2011; Escobedo, Flaquer \& Navarro, 2012; Salazar, 2013).

La construcción social de la maternidad y su articulación institucional con las políticas sociales ha sido extensamente estudiada sobre todo desde la década de los años noventa del siglo pasado (Leira, 1992; Lewis, 1992, ed 1997 y 2006; Orloff, 1993, 1996 y 2009; Pfau-Effinger, 2004 y 2005). El papel de los padres como 
proveedores económicos se ha dado históricamente por sentado y sólo recientemente se están llevando a cabo investigaciones sobre su contribución a las familias como cuidadores. Si bien el modelo familiar del sustentador universal se ha convertido en eje referencial de las políticas familiares y sociales actuales, todavía hay una clara ambivalencia en torno a las medidas aplicadas para lograr la igualdad de oportunidades entre las madres y los padres. Con la excepción de los países nórdicos, hay escasas evidencias de convergencia hacia un modelo de familia basado en dos sustentadores a tiempo completo por más que se hayan registrado avances significativos hacia una dedicación más equilibrada y menos desigualitaria de hombres y mujeres al trabajo remunerado. El análisis empírico revela un importante progreso hacia la individualización en la medida en que las políticas sociales promueven y valorizan la agencia y la autosuficiencia individual y externalizan fuera de la familia una parte de la atención a la infancia. Sin embargo, también se advierte la persistencia de un renovado familiarismo. Más que un avance inequívoco hacia un modelo de trabajador individualizado, se está fomentando una familia de doble sustentador con una cierta especialización de género, que estaría a mitad de camino entre las antiguas dependencias y la nueva 'independencia.' (Lewis et al, 2008; Daly, 2011). Así, en general, las madres acceden en mayor medida y con relativa mayor facilidad que los padres al uso de las licencias parentales remuneradas o a la posibilidad de adaptar horarios de trabajo flexibles, en interés de los hijos, excepto en el sector público donde la cultura laboral promueve una mayor igualdad de género.

Si bien es cierto que las responsabilidades familiares paternas en el ámbito de la parentalidad se reconocen cada vez más en la mayoría de los países, la transición hacia el nuevo modelo de sustentador (y cuidador) universal requiere una reorganización fundamental de los Estados de bienestar. Sólo en la medida en que se capacite y promueva la participación de los hombres en el cuidado no remunerado en un nivel similar al de las mujeres, sobre todo a partir de la crianza de los hijos pequeños, se podrá mejorar la igualdad de género y la calidad de los vínculos entre padres e hijos, posibilitando la construcción de una nueva paternidad. La introducción y extensión de cuotas paternas en los sistemas de licencias parentales y la monitorización de su uso por parte de los padres se ha convertido en un referente simbólico, como indicador de este proceso y medida de política social ampliamente reconocida y estimulada en el ámbito de la Unión Europea (Leira, 1998; Kamerman \& Moss, 2009). Los resultados de diversas investigaciones han mostrado ya evidencias de que las licencias paternales contribuyen a un cambio decisivo en el desarrollo de unas nuevas pautas de paternidad (Duvander \& Jans, 2008; O’Brien, 2009; Brandth \& Kvande, 2009).

En el año 2007, en España se amplió y reformuló el sistema de licencias parentales, y en particular el permiso de paternidad, en el marco de una ley orgánica de igualdad de oportunidades entre hombres y mujeres (Escobedo, 2008). Reuniendo resultados de diversas investigaciones realizadas desde entonces (Escobedo, Navarro y Flaquer, 2008; Escobedo, 2009; Escobedo, Flaquer y Navarro, 2012), el presente artículo se suma al proceso de evaluación del sistema español de licencias parentales, desde la perspectiva de su contribución relativa a la construcción 
de unas nuevas paternidades y a una nueva política social de la paternidad en nuestro país.

\section{El impacto diferencial de la paternidad y la maternidad sobre el empleo}

El nacimiento de un primogénito constituye una encrucijada en la trayectoria laboral de cada individuo, en la que padres y madres toman decisiones en un contexto de condicionantes institucionales e itinerarios social y culturalmente modelados de forma distinta para ambos sexos. Esta encrucijada se expresa y resuelve de forma diversa en los países de la Unión Europea, según sus tradiciones de política social, de regulación laboral y en materia de servicios de atención y educación infantil.

El tránsito a la paternidad se asocia con la consolidación del estatus profesional del hombre y de su rol de proveedor de recursos salariales al hogar. En cambio, el acceso a la maternidad se asocia con una intensificación del rol de cuidadora de la mujer en el ámbito doméstico que puede afectar en menor o en mayor medida a la esfera profesional, de forma coyuntural o estructural. Esta especialización de roles en términos de género tiende a producirse tras el nacimiento de un primer hijo y durante los primeros años de vida de éste hasta que se normaliza la participación de los pequeños en la educación infantil y se supera la fase de pequeñas enfermedades frecuentes propias del proceso de maduración de los niños. La afectación temporal de la dedicación de la mujer al empleo depende fuertemente de la calidad y las políticas de servicios de atención y educación infantil, pero también de la implicación paterna en las tareas de cuidado. Lo cual también depende del nivel educativo de la madre: a mayor nivel educativo los diferenciales de género se reducen en intensidad y en duración.

Tomando como referencia la Encuesta Europea de Condiciones de Vida de 2006, en el conjunto de la Unión Europea la mitad de los menores de tres años son cuidados en casa de forma exclusiva por sus progenitores. La atención parental es básicamente materna y dominante durante el primer año, o año y medio de vida, de forma más o menos protegida mediante los sistemas nacionales de licencias maternales y parentales (Escobedo, 2009). Por otro lado, una cuarta parte de los menores de tres años reciben una atención y educación infantil formalizada, con proporciones que oscilan entre tres cuartas partes de los menores de 3 años en Dinamarca y menos del 5\% en países como la República Checa o Polonia (Eurostat, datos 2005-2010). La cuarta parte restante de estos niños pequeños son atendidos mediante arreglos informales -canguros o asistentas retribuidas- o bien mediante ayuda familiar no retribuida. En España la atención parental exclusiva es menor (41\% en 2006), mientras que la cobertura de los servicios infantiles formales asciende en torno a 4 de cada 10 menores de 3 años, por encima de la media de la UE, aunque esta proporción presenta una gran variabilidad por Comunidades Autónomas, como efecto de la diversa regulación y financiación pública de estos servicios. 
La reducción de la dedicación al empleo se instrumenta de forma diversa: licencias más o menos retribuidas que protegen un tiempo de cuidado, en el contexto del empleo, pautando la reincorporación al trabajo; reducciones voluntarias y reversibles de la jornada de trabajo, o involuntarias si se producen en un contexto de falta de elección e irreversibilidad; episodios de desempleo remunerado; o bien finalmente mediante la inactividad, es decir mediante la exclusión del sistema contributivo de protección social.

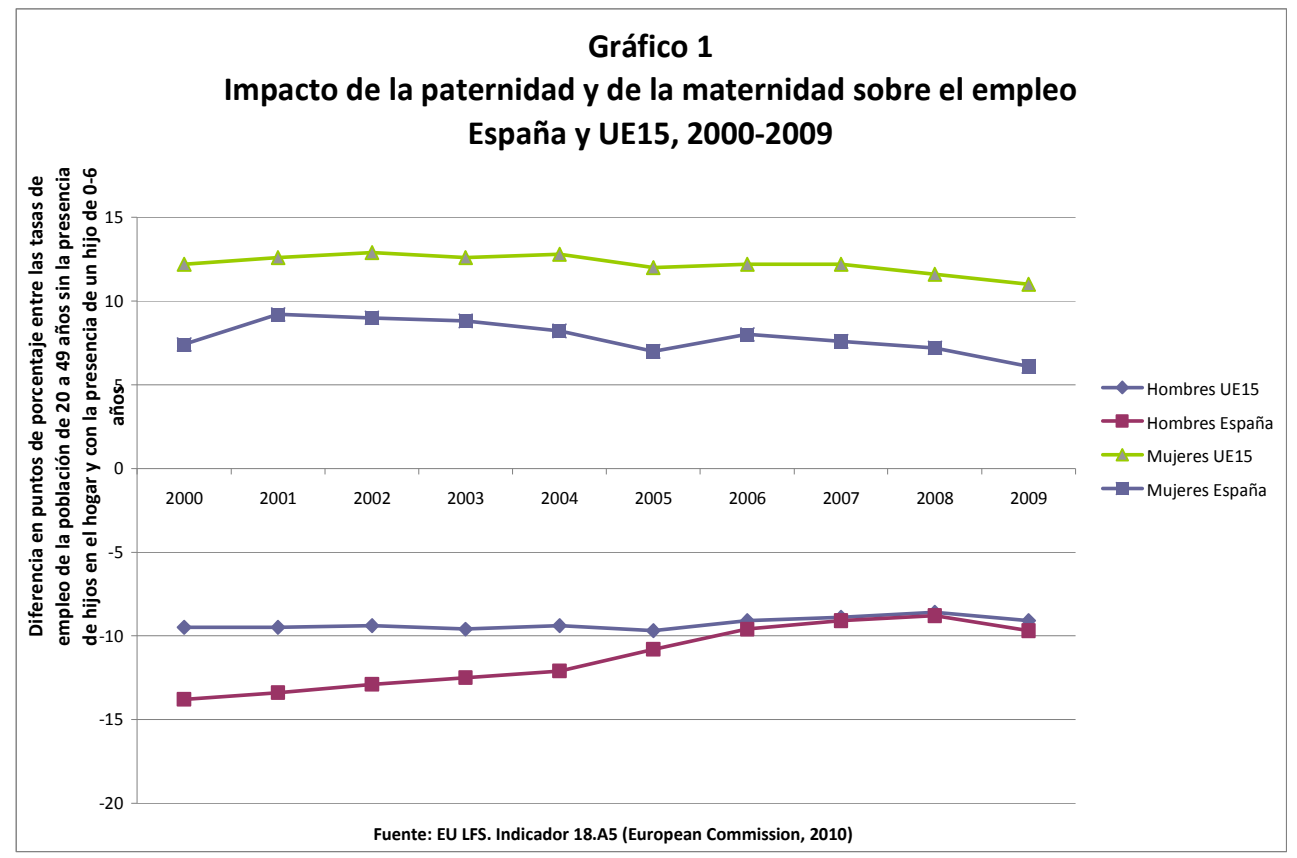

Utilizando el Indicador 18.A5, extraído del compendio de indicadores para hacer un seguimiento de las directrices de empleo elaborados por la Comisión Europea con datos de la Encuesta Europea de Población Activa (EU-LFS) se puede estimar el impacto de la maternidad y de la paternidad sobre las tasas de empleo ${ }^{1}$. Según se puede observar en el Gráfico 1, ese impacto no sólo es distinto para hombres y mujeres sino que las diferencias entre España y la media europea son bastante significativas. Mientras que en el período considerado 2000-2009 se produce una convergencia entre España y la media de la UE15 en relación con el indicador sobre el impacto de la paternidad, cuya tendencia refleja en España una cierta reducción

${ }^{1}$ Diferencia en puntos de porcentaje entre las tasas de empleo de la población de 20 a 49 años sin la presencia de hijos en el hogar y con la presencia de un hijo de 0-6 años (Indicators for Monitoring the Employment Guidelines. Compendium 2010). 
de la intensificación del empleo masculino tras el nacimiento del primer hijo, las mujeres españolas que acceden a la maternidad muestran una tasa de interrupción del empleo muy inferior a la media de las madres europeas. La divergencia entre las madres españolas y europeas se mantiene, con algunas variaciones, a lo largo de la década (European Commission, 2010).

Tomando como referencia el año 2006, antes del impacto de la actual crisis económica, mientras que los padres intensifican sus tasas de ocupación sin excepción en el conjunto de la Unión Europea en diez puntos porcentuales en promedio, las madres con hijos pequeños tienen comportamientos más variables según las prestaciones sociales disponibles y la calidad del empleo. Así el impacto de la maternidad sobre la tasa de empleo femenina oscila entre un aumento de 5 puntos porcentuales ${ }^{2}$ y un descenso de 40 p.p., situándose la media europea en un descenso de 14 p.p. en 2006 (y de 8,4 p.p. en el caso español). El descenso de las tasas de empleo maternas se duplica entre un primer y un segundo hijo (de -8,6 a 16,2 p.p. respecto a las mujeres sin hijos), y se triplica en familias numerosas (-32 p.p.), aunque la variable más relevante es el nivel educativo de la madre ya que el diferencial entre las tasas de empleo de las madres con niveles superiores e inferiores de educación se sitúa en 37 p.p. en presencia de menores de 6 años en el hogar. Por otro lado, cuando el hijo menor supera los 6 años el impacto de la maternidad en las tasas de empleo se reduce notablemente en el conjunto de la Unión Europea, y sólo permanece significativo en familias numerosas y en hogares cuyas madres tienen niveles educativos bajos (Escobedo, Navarro y Flaquer, 2008).

La posición relativa de España se puede sintetizar como la de un país en que la generalización del empleo femenino y materno se ha producido más recientemente, con un menor diferencial que en otros países para las madres que logran mantenerse en el sistema del empleo, respecto a las mujeres sin hijos, en particular para las mujeres con niveles formativos más altos. La edad de los hijos influye poco, siendo las tasas de empleo maternas similares con hijos pequeños o con hijos a partir de 6 años, excepto en familias numerosas en que el empleo materno se recupera incluso por encima de las familias con dos hijos. Las tasas de empleo paternas son en 2006 ligeramente superiores a la media de la UE, en particular para los hombres con niveles educativos bajos. En España, antes de la crisis, la variable educativa masculina afectaba en menor medida las tasas de empleo paternas que en el conjunto de la Unión Europea (4 p.p. entre padres con niveles superiores e inferiores de educación, respecto a 11 p.p. en la UE).

En el caso español, la observación longitudinal durante el periodo 2003-06 realizada mediante el análisis de la Muestra Continua de Vidas Laborales de la Seguridad Social (MCVL_2006) nos ha permitido contrastar comportamientos laborales claramente divergentes entre hombres y mujeres, que tuvieron un primogénito en el año 2003, tanto respecto al riesgo de interrupción temporal no

\footnotetext{
${ }^{2}$ De ahora en adelante, p.p.
} 
regulada del empleo (es decir, sin recurrir a la excedencia por cuidado de hijos/as) como a la reducción del tiempo de trabajo. Sólo el $17 \%$ de los hombres y $8 \%$ de las mujeres de la muestra analizada no presentan ningún impacto sobre su carrera de cotización laboral tras el nacimiento de un primer hijo/a en 2003. Para dos de cada tres hombres (66\%) que han cambiado de estado laboral (entre las categorías de asalariado, autónomo, desempleo contributivo, inclusión/exclusión del sistema contributivo de seguridad social) entre 2003-06 el impacto de los hijos/as puede definirse como positivo en términos del empleo y cotización social, mientras que para las mujeres este porcentaje se reduce al $41 \%$.

Las diferencias entre padres y madres alcanzan su punto álgido en torno al primer año de vida de los hijos/as. En ese momento del tiempo, las mujeres asalariadas se reducen al $37 \%$ de nuestra submuestra de primo-progenitoras, $31 \%$ salen del sistema contributivo de Seguridad Social, y 17\% permanecen afiliadas bajo prestaciones por desempleo, mientras que para los hombres estas categorías representan el $62 \%, 12 \%$ y $8 \%$ respectivamente. Si bien las tendencias observadas en el comportamiento laboral de las mujeres consiguen invertirse nuevamente, en ningún caso vuelven a alcanzar los valores iniciales. El uso de la excedencia por cuidado de hijos/as no consigue neutralizar el impacto de la maternidad o de la paternidad, en la medida en que su valor máximo no alcanza el $2 \%$ de los casos en ningún momento, y mayoritariamente la reducción de tiempo de trabajo se instrumenta mediante nueva contratación y no mediante el mecanismo de reducción de tiempo de trabajo protegida y reversible dentro de la misma relación laboral en el período analizado (Escobedo, Navarro y Flaquer, 2008).

Entendiendo que las políticas de conciliación de la vida laboral y familiar se orientan a compensar los impactos adversos de la maternidad y la paternidad en las carreras laborales y de protección social, estos diferenciales pueden interpretarse como un déficit de nuestras políticas sociales, tanto en relación al impacto adverso de la maternidad sobre el empleo como al escaso uso de excedencias parentales (a tiempo parcial o tiempo completo) que se observa tanto para hombres como para mujeres.

\section{Permisos y licencias de paternidad}

Las políticas de licencias parentales surgieron como un mecanismo para proteger la formación familiar y la crianza de los niños en sociedades donde se generalizaba el trabajo materno. Hungría fue el país pionero introduciendo en 1967 una licencia larga para las madres, tras la licencia por maternidad, dada la escasez y costes de los servicios de atención infantil para los niños más pequeños. La licencia parental, definida como permiso laboral por cuidado de hijos cuyo ámbito de aplicación se ampliaba a los hombres, se introdujo en Suecia en 1974. A partir de entonces se desarrollan en Europa occidental unos sistemas de licencias parentales y por cuidados de familiares progresivamente más individualizados y mejor retribuidos, como mecanismos facilitadores de la articulación entre familia y empleo, en sociedades tendentes al modelo familiar del doble sustentador. Las licencias 
parentales y para los cuidadores informales se han convertido en un mecanismo regulador de la relación dinámica entre los individuos organizados en familias, el mercado de trabajo y el Estado. Representan un encaje clave en la construcción institucional de un nuevo modelo de ciudadanía activa basada en el ejercicio simultáneo del derecho y el deber de trabajar y de cuidar (universal adult worker and care giver citizen model), sobre la base del cual se erige el sistema familiar más diverso de las sociedades postindustriales (Escobedo, 2013).

Las licencias parentales y por cuidado de familiares son formas reguladas de ausencia del lugar de trabajo, o de interrupción de las prestaciones laborales habituales, asociadas a prestaciones económicas (retribuciones substitutivas del salario y otros derechos de protección social) para atender al nacimiento y crianza de los hijos muy pequeños o al cuidado de adultos en situación de enfermedad, accidente o severa dependencia. Tienen por objeto proteger simultáneamente y en diferentes grados los intereses de los niños, de las madres y de los padres empleados, de las personas dependientes y sus cuidadores, de los empresarios así como otros intereses sociales (normalmente representados por las instituciones públicas en sus distintas actividades: sanidad, educación, protección social).

Las licencias parentales y por cuidado de familiares son principalmente: la licencia por maternidad, la licencia por paternidad, la licencia parental (en España instrumentada a tiempo completo como excedencia por cuidado de hijos y a tiempo parcial como reducción de la jornada laboral por guarda de hijos) y los permisos para cuidar de un hijo o familiar enfermo.

Las licencias parentales y por cuidado de familiares se enmarcan en la categoría más amplia de los sistemas de interrupción de carrera (por motivos familiares, de formación, de salud u otros) y constituyen un instrumento para redistribuir el tiempo de trabajo y el tiempo de cuidado a lo largo del ciclo vital de los individuos, con la finalidad ideal de conseguir una organización del trabajo y del tiempo más sostenible para la calidad de vida y para la cohesión social. Son también un elemento clave para comprender la lógica de funcionamiento de los sistemas de atención a la primera infancia en los diferentes países.

Las licencias parentales contribuyen a la conformación e institucionalización de nuevas trayectorias tanto de maternidad como de paternidad, en un contexto de parentalidad tendente a desarrollarse de manera más planificada, negociada y reflexiva. Comportan la definición y la legitimación social de unos períodos a la vez protegidos y limitados en el tiempo consagrados a la crianza de los hijos más pequeños. Históricamente estos períodos se han definido y construido socialmente de maneras claramente distintas para las mujeres y para los hombres. Sin embargo, las nuevas tendencias en las políticas de licencias parentales sugieren el desarrollo de un tipo de sistemas de licencias (a modo de recursos integrados de tiempo regulados por los sistemas públicos de seguridad social) más neutros desde el punto de vista del género, individualizados y flexibles, que se articulan a la regulación pública del desarrollo de los servicios de atención y educación infantil (Deven \& Moss, 1999; Kamerman \& Moss, 2009). Estos desarrollos institucionales abren la posibilidad de nuevas elecciones individuales y una manera más reflexiva de 
plantear la parentalidad y el cuidado informal en el ámbito familiar y doméstico, tanto para las mujeres como para los hombres.

O’Brien (2009) ha analizado cómo la licencia parental impulsa la inversión emocional paterna y desencadena su conexión física y emocional con sus hijos desde el nacimiento. Así, sugiere que se está generando un nuevo eje de polarización sobre los niños: el riesgo de nacer ya sea en un hogar o en un país ricos o pobres en tiempo y licencias parentales de calidad. Este enfoque explicaría el mayor riesgo de los padres, en relación con las madres, de experimentar la pérdida de contacto con sus hijos tras el divorcio, como resultado de sus menores inversiones previas en tiempo de cuidado parental físico directo, con todas las graves consecuencias, tanto de índole material como emocional, que ello comporta para los menores (MacLanahan, Tach \& Schneider, 2013).

La licencia parental constituye un mecanismo de política social concebido para contrarrestar los efectos económicos adversos, que se producirían en ausencia de estas medidas en familias de doble sustentador. Por un lado, protegen la vinculación de las madres con el mercado de trabajo y su trayectoria laboral, sin perjuicio de su rol cuidador $\mathrm{y}$, por otro, fomentan la participación de los padres en la crianza de los hijos, sin perjuicio de su rol proveedor económico. A través de la investigación internacional comparada (Thévenon, 2011), se ha podido conocer mejor cómo los sistemas de licencias parentales eficaces (es decir los que son utilizados ampliamente) permiten paliar estos tipos de riesgos sociales, siempre que garanticen la seguridad del empleo, la estabilidad económica, el uso compartido de estas medidas entre hombres y mujeres y la individualización de los vínculos parentales hacia los hijos. Grupos y movimientos masculinos han apoyado en particular la individualización de los derechos del padre a gozar de las licencias paternales (sin que sea un derecho derivado de la situación laboral o de la elección de la madre) como fundamento de su vínculo paterno y del desarrollo de una relación emocional y físicamente activa y presente con sus hijos, participando desde su nacimiento, en unas rutinas de cuidado, que además del valor que tienen en sí mismas, logren prevenir o mitigar los efectos de la ruptura conyugal, si acontece más tarde, preservando así los contactos entre padres e hijos. De forma consistente, diversas investigaciones han evidenciado que las políticas de licencias parentales que promueven una paternidad activa en el ámbito de los cuidados físicos han contribuido a modificar de forma estructural y en una orientación más igualitaria la distribución de funciones y tareas entre madres y padres, en distintos países del norte de Europa (Rostgaard, 2002; Lammi-Taskula, 2006; O'Brien, Brandth \& Kvande, 2007; Moss, 2008). Recientemente este efecto se ha contrastado también en el ámbito español (Meil, 2013, Meil y Rogero, en este monográfico) y se ha comprobado cómo los padres que hacen uso de las licencias parentales (en igualdad de circunstancias, incluyendo las preferencias igualitarias) dedican a sus hijos una atención de calidad durante más tiempo.

En la década de 1990, la licencia parental (englobando madres y padres) se extiende por Europa con una creciente atención en promover un uso más igualitario entre hombres y mujeres, a partir de la constatación de que los sistemas puestos en 
marcha en la década de los 1980, más orientados a las madres, no habían corregido sino más bien consagrado diferenciales de género propios del modelo de sustentador masculino principal, con impactos estructurales posteriores en las trayectorias laborales maternas. Tras descubrir que los padres prácticamente no utilizaban las licencias parentales, en la medida que eran reguladas como derechos familiares transferibles entre miembros de la pareja, Noruega introdujo en 1993 un período de licencia parental de cuatro semanas individualizado para el padre, que por tanto se perdía si el padre no hacía uso de él y que pasó a denominarse la cuota del padre ("father's quota" o "daddy leave") (Leira, 1998). Ante el rotundo éxito de la medida que indujo automáticamente a los padres a utilizar la práctica totalidad de su período, Suecia también introdujo en su regulación social este mecanismo en 1995, con resultados parecidos. En 2000, Islandia reformuló su sistema de licencias parentales, siguiendo un modelo $3+3+3$, donde tres meses son para la madre, tres para el padre, siendo los tres meses restantes utilizados como desee cada familia ${ }^{3}$. En los tres casos mencionados, se logró casi automáticamente el aumento del uso paterno de la licencia parental, en la misma proporción en que se aumentaba la cuota paterna intransferible. Reflejando la proporción de cuotas paternas en estos países, en 2007 el porcentaje total de días con prestación económica en concepto de licencia parental utilizadas por los padres fue de $31 \%$ en Islandia, el $22 \%$ en Suecia, el $11 \%$ en Noruega y el $6 \%$ en Dinamarca y Finlandia (Haataja, 2009).

A raíz de la expansión de métodos de evaluación política comparada (siguiendo la senda del método abierto de coordinación iniciado en la Estrategia Europea por el Empleo), en la primera década del siglo XXI la mayoría de países de la UE han revisado y monitorizado sus políticas de licencias parentales. Las cuotas paternas no transferibles de licencia parental se están extendiendo entre los países, ya sea siguiendo una pauta de "utilice o pierda" ("use-it or lose-it") esa parte de la licencia, ya sea conceptualizadas como una bonificación extra para la familia. Por ejemplo, en Finlandia, en Alemania (desde 2007) y más recientemente en Portugal (desde 2010), si el padre utiliza una parte de la licencia, se compensa a la familia alargando el tiempo global de licencia parental disponible. También la licencia de paternidad, con ocasión del nacimiento de un hijo, se está extendiendo a un mayor número de países. Como actualmente no está regulada en la Unión Europea, en 2010 el Parlamento Europeo propuso establecer un mínimo de dos semanas de permiso de paternidad pagado en su totalidad, en el marco de la discusión de una nueva Directiva sobre el permiso de maternidad (Enmienda 125 a la posición del Parlamento Europeo, aprobada el 20/10/2010), cuya implementación ha quedado sin embargo en suspenso en el contexto de la crisis. La investigación ha hecho hincapié en el interés de la licencia de paternidad para apoyar una presencia más

${ }^{3}$ En diciembre de 2012 el Parlamento Islandés ha aprobado ampliar el sistema a 12 meses entre 2013 y 2016 con una distribución 5+5+2 (cinco meses exclusivos para las madres y para los padres, y los dos restantes transferibles entre ambos). 
activa de los padres en el nacimiento, contribuyendo así a modificar prácticas muy matrifocales en maternidades y en las políticas de salud, que relegan a un segundo plano la paternidad (Truc, 2006).

En 2010, veinte de los Estados miembros de la UE tenían un permiso de paternidad regulado, de entre 2 y 90 días laborales, asociados a compensaciones que oscilaban entre la compensación total del salario por parte de la Seguridad Social o la ausencia total de compensación. Las tasas de utilización de los permisos de paternidad son relativamente altas, con más de dos tercios de los padres por lo general tomando tales disposiciones cuando están disponibles (Moss, 2013).

Todo ello ha generado un debate que se ha extendido globalmente sobre el potencial y límites de este tipo de medidas y la importancia de su diseño específico, que ha influido en cambios legislativos acaecidos posteriormente en un buen número de países y difundido a organizaciones internacionales más allá de la Unión Europea, como la OCDE (2011), la misma organización de Naciones Unidas a través de la OIT o de los trabajos preparatorios del $20^{\circ}$ aniversario de la celebración del Año Internacional de la Familia en 1994 y que en 2014 tendrá como ejes el equilibrio entre familia y trabajo, la pobreza infantil y la solidaridad intergeneracional (O’Brien, 2012).

\section{El sistema español de excedencias y prestaciones para el cuidado de hijos y familiares}

Reformulado durante la transición democrática en el contexto del Estatuto de los Trabajadores, en España el sistema de licencias parentales pivotó entre 1980 y 2007 sobre un permiso de maternidad bien retribuido y un conjunto de medidas no retribuidas y reguladas por igual para hombres y mujeres (la excedencia por cuidado de hijos de hasta tres años o las reducciones de jornada por guarda de un menor de 6 años), poco utilizadas por las mujeres y todavía menos por los hombres. En lo que respecta a las políticas de articulación entre empleo y familia, en España se ha producido un mayor consenso en torno al desarrollo de la educación infantil y los servicios infantiles para niños en edad preescolar (con notables divergencias sobre los criterios de calidad y los compromisos de financiación pública según Comunidades Autónomas) que en torno a reformular un modelo eficaz y moderno de licencias parentales.

En un ejercicio de modelización de políticas de licencias parentales, España se incluye en una tipología de licencias parentales retribuidas cortas ('the short-leave modified 'male-breadwinner' model' junto con Italia y Grecia), congruente con su adscripción a un régimen de bienestar familiarista, que ofrecen un período relativamente breve de licencia parental bien retribuida, basándose en el supuesto de la persistencia de una proporción de hogares en que todavía se aplica el patrón del sustentador masculino o de un cuidado significativo de los niños por parte de la familia extensa (Wall \& Escobedo, 2009 y 2013). Desde los años ochenta en España el énfasis de los discursos se ha centrado de manera congruente en la 
integración femenina en el mercado laboral, con relativa poca atención cuando no oposición a las políticas de licencias, que son percibidas como una amenaza para la integración igualitaria de las mujeres en el ámbito laboral. Existe un relativo consenso entre partidos políticos y agentes sociales sobre el interés en promover los servicios infantiles como principal instrumento de conciliación de la vida laboral y familiar, ya que tienen la ventaja de no interferir en las dinámicas laborales y entroncan con las políticas educativas, más sólidas en la agenda política española que las políticas familiares. Por otro lado resultan poco visibles los grupos que demandan unas licencias retribuidas más largas desde perspectivas sanitarias y de calidad de vida, para facilitar la transición a la educación infantil cuando realmente es socialmente más accesible, es decir a partir del primer año.

El interés por las licencias parentales emerge a partir de 1999, cuando el concepto de conciliación de la vida laboral y familiar se populariza en la agenda política española, siguiendo el impulso de las directivas europeas, la Estrategia Europea de Empleo y los Planes de Igualdad de Género, en un período de clara expansión del empleo femenino y materno. Ante la observación de que las licencias parentales no remuneradas resultan inasequibles, y empezando por Navarra en el año 2000 en un contexto de bonanza económica, varias comunidades autónomas introducen prestaciones económicas complementarias a las licencias no retribuidas (que oscilaban entre un tercio a las cuatro quintas partes en algunos casos del salario mínimo, siendo éste de $645 €$ en 2013) para estimular el uso de la excedencia por cuidado de hijos y de la reducción del tiempo de trabajo por guarda de hijos. Por otro lado, se va extendiendo una retórica igualitaria, en la que se desarrollan medidas que aparentemente estarían orientadas al mayor uso por parte de los hombres, como la posibilidad de que la madre transfiera una parte creciente de su licencia y prestación de maternidad al padre ${ }^{4} \mathrm{o}$ en algunas comunidades autónomas el establecimiento de pequeños incentivos económicos, que en la práctica tienen una incidencia mínima ya que son tipos de medidas que, al no tratarse ni de cuotas intransferibles ni de retribuciones substitutivas del salario, han sido descartadas ya en muchos países por no fundamentar el uso masculino de licencias.

En cambio, sí tienen un impacto significativo en la reducción del diferencial de género medidas que se aplican en el sector público, sin pérdida retributiva. En 2002, la Generalitat de Cataluña introduce una serie de medidas restringidas al sector público, que amplían algunas licencias, como el permiso por nacimiento de un hijo e incentivan de forma total o parcialmente remunerada las reducciones de la jornada laboral por guarda de hijos. El análisis de los datos sobre el uso de esas medidas complementarias en el sector público catalán entre 2003-2006 muestra un uso generalizado por parte de los padres de la reducción de un tercio del tiempo de

${ }^{4}$ En 1999 se amplia de 4 a 10 semanas la posibilidad para la madre de transferir al padre una parte de su prestación por maternidad, vigente desde 1989. 
trabajo (sin pérdida retributiva) durante el primer año del bebé y un uso notable -aunque mayor por parte de las mujeres- de las reducciones de jornada de un tercio (con $80 \%$ del salario) o de media jornada (con 60\% del salario) (Escobedo y Navarro, 2007). En el contexto laboral favorable de un sector público en expansión en esos años (con retribución y cultura laboral favorable al uso de las medidas) tanto el uso masculino como femenino observado ha sido notablemente alto. Las modalidades de licencias a tiempo parcial han resultado un buen referente de tipos de medidas de buena calidad, voluntarias y reversibles, y más acordes con la cultura laboral de nuestro país, en la que la desvinculación total del lugar de trabajo (en el caso de la excedencia) es percibida como mucho más arriesgada para el trabajador.

En 2007 el sistema español de licencias parentales se reformula en el marco de una Ley de Igualdad de Género (Ley Orgánica 3/2007, para la Igualdad Efectiva de Mujeres y Hombres), que traslada el foco de las políticas de licencia parental hacia la paternidad y hacia un sistema de reducciones del tiempo de trabajo voluntario y reversible muy flexibles y que se extiende también a contingencias de cuidado de adultos. No obstante, desde la perspectiva de las políticas de atención a la primera infancia se continúa haciendo énfasis en la expansión de la educación infantil.

La Ley Orgánica 3/2007, para la Igualdad Efectiva de Mujeres y Hombres, introduce un nuevo permiso de paternidad (muy parecido al que se aprobó en Francia en 2002). Es la primera vez que en España se concede a los padres un derecho individualizado y financiado por el sistema de Seguridad Social, ya que la retribución de los dos días de permiso de los padres en razón del nacimiento de sus hijos anteriormente existentes va a cargo de los empleadores (de hecho, este breve permiso por nacimiento existe desde los años treinta) (Escobedo, 2009). La ley de 2007 incluía el compromiso de prolongar el permiso de paternidad a cuatro semanas, a partir del $1^{\circ}$ enero de 2011, pero la medida se ha pospuesto debido a las restricciones presupuestarias causadas por la crisis financiera. La Ley Orgánica 3/2007 supuso también la reformulación de la Ley 7/2007, del Estatuto Básico de Empleado Público (EBEP) que incorpora mejoras y algunas de las disposiciones que desde el año 2005 ya había introducido de modo experimental el Plan Concilia para los empleados públicos estatales.

La duración del permiso de paternidad es actualmente de quince días, remunerados al cien por ciento, por la Seguridad Social en las mismas condiciones que la prestación por maternidad, a excepción de los dos primeros días que siguen financiando los empleadores en el sector privado. La regulación del permiso por nacimiento se ha redactado en términos de género neutros a fin de integrar los casos de parejas de progenitores del mismo sexo.

Al mismo tiempo la ley amplía y flexibiliza las opciones de reducción del tiempo de trabajo por guarda de hijos entre un octavo (antes un tercio) y la mitad del tiempo de trabajo ordinario, extendiéndose hasta que el niño cumple 8 años (o 12 en el sector público, antes hasta los 6 años) o para cuidar a un familiar dependiente. 
Tras las experiencias nórdicas -y en particular la islandesa-, ampliamente difundidas en las redes globales de intercambio de conocimiento, con un papel destacado de la Red de Expertos en Licencias Parentales (Moss, 2008, 2013; Kamerman \& Moss, 2009), diversos grupos feministas y a favor de la igualdad de género organizan una Plataforma para promover Permisos Iguales e Intransferibles por Nacimiento y Adopción y proponen un sistema de licencias parentales iguales e intransferibles retribuidas al $100 \%$ del salario para hombres y mujeres como una estrategia para promover la participación masculina en el cuidado de niños y para contrarrestar la estigmatización de las mujeres en el mercado de trabajo (Castro y Pazos, 2008) ${ }^{5}$. Movimientos de hombres en favor de las nuevas masculinidades o que promueven la custodia compartida después del divorcio se suman a las demandas de licencias propias e intransferibles para los padres, y de una mayor implicación de los hombres en los cuidados de los hijos desde el nacimiento ${ }^{6}$.

Actualmente, en el contexto de crisis económica, cabe destacar que si bien se han retirado algunas de las ayudas por nacimiento de hijos o hijos a cargo, estas medidas estatales se han mantenido, lo cual es importante en un contexto en que el empleo femenino apenas ha disminuido (-4 p.p.) a pesar de la crisis que sí está afectando mucho más al empleo masculino (-16 p.p.), en el grupo de edad entre 25 y 54 años (datos INE-EPA, 1er trimestre, entre 2007 y 2012). Sin embargo, una parte de las medidas adoptadas por las comunidades autónomas han sido reducidas o eliminadas (Escobedo y Meil, 2013). Una de las reformas más destacadas, desde el punto de vista del impacto notable que tenía en la igualdad de uso entre padres y madres, es la eliminación a mediados de 2012 de la reducción del tercio de tiempo de trabajo retribuido en el sector público catalán, que había tenido resultados tan positivos entre los varones. Si bien se mantienen las reducciones incentivadas con menor pérdida retributiva, estas sí producen un sesgo en el uso diferencial entre hombres y mujeres.

${ }^{5}$ Véanse, a ese respecto, las propuestas radicales y la destacable tarea de lobby realizadas por la Plataforma por permisos iguales e intransferibles por nacimiento o adopción (PPINA) (2005-13) tanto a nivel español como internacional (http://www.igualeseintransferibles.org/).

${ }^{6}$ En este sentido, cabe señalar la reciente constitución en agosto de 2013 en Bonn (Alemania), con participantes de 14 países europeos y de América del Norte, de la International Platform on Shared Parenting (http://www.twohomes.org) sobre el fomento de la custodia compartida, con un importante énfasis en la implicación paterna, en el equilibrio de género y en el bienestar infantil. 


\section{Análisis empírico de datos sobre el uso de licencias de paternidad y de maternidad percibidas por el padre}

Los análisis empíricos realizados en los últimos años sobre el uso de licencias parentales en España se basan fundamentalmente en datos de encuestas ${ }^{7}$. En este sentido, disponemos de valiosas aportaciones de los resultados de diversas investigaciones sobre distintos aspectos del uso de los permisos, tanto por parte de hombres como de mujeres (Meil, 2011, 2013; Escot \& Fernández-Cornejo, 2012; Lapuerta, Baizán \& González, 2011; Escobedo, Navarro \& Flaquer, 2007 y 2008; Escobedo \& Navarro, 2007). El enfoque que proponemos aquí es mejorar el aprovechamiento de los datos publicados regularmente sobre el número de prestaciones de paternidad y de maternidad percibidas por el padre concedidas anualmente con el fin de monitorizar su evolución en el tiempo y su distribución territorial según comunidades autónomas, así como a través de un análisis de correlaciones explorar posibles factores socioestructurales que inciden en el uso de los permisos ${ }^{8}$. Esta metodología requiere como paso previo la construcción de algún indicador en forma de tasa que permita estimar de forma relativa los niveles de uso de los permisos en distintos años y unidades territoriales.

Los datos analizados en esta parte empírica se han descargado del Anuario de Estadísticas del Ministerio de Empleo y Seguridad Social 2011, que juntamente con otras informaciones sociolaborales recopila y publica anualmente el Ministerio. Las informaciones sobre prestaciones por maternidad y paternidad proceden de la Subdirección General de Gestión de Incapacidad Temporal y Otras Prestaciones a Corto Plazo del Instituto Nacional de la Seguridad Social, entidad gestora responsable de la gestión de estas prestaciones. Desde 2007 se publican datos sobre

${ }^{7}$ La puesta en marcha de la Muestra Continua de Vidas Laborales en 2006 ha significado un gran estímulo para el análisis de las excedencias y reducciones horarias, aunque tiene la limitación de no incorporar en la misma base de datos las licencias de maternidad o paternidad. Se trata además de una base de datos compleja de manejar. Los módulos sobre conciliación de la EPA (2005 y 2010) desarrollados por el INE en coordinación con Eurostat, son también una interesante fuente de información, aunque limitada en relación con la comprensión del uso de permisos. Las últimas investigaciones con encuestas específicas o con trabajos de campo cualitativos en el marco de proyectos de investigación, realizadas por distintos equipos, van a permitir profundizar en los modelos explicativos del comportamiento individual en nuestro país. Sin embargo, nos parece asímismo importante establecer metodologías de seguimiento de la evolución temporal de las percepciones, particularmente en un contexto económico y laboral tan cambiante como el actual.

${ }^{8}$ Aunque los datos publicados en el Anuario de Estadísticas del Ministerio de Empleo y Seguridad Social se refieren en sentido estricto a prestaciones concedidas, en el texto de esta sección, para evitar forzar en demasía el empleo del lenguaje, utilizamos indistintamente los términos de permisos o licencias. 
el número de prestaciones de paternidad y su coste en miles de euros por provincias y comunidades autónomas. En nuestro análisis nos hemos centrado en el período 2008-2011, ya que los datos correspondientes a 2007 no cubren todo el año. En esta explotación nos hemos limitado al análisis empírico de datos sobre prestaciones de paternidad y de maternidad percibidas por el padre al ser más adecuadas para la obtención de tasas de uso que permitan estimar la magnitud del fenómeno desde un punto de vista relativo.

\subsection{Construcción de un indicador para estimar el uso de los permisos de paternidad y de maternidad disfrutados por padres}

Una de las restricciones que impone la investigación con datos sobre prestaciones es que, antes de proceder a su análisis, conviene elaborar algún tipo de indicador que permita estudiar adecuadamente su uso. Si bien el uso de las prestaciones de maternidad se puede estimar fácilmente a base de relacionar el número de prestaciones concedidas con los hijos nacidos durante el año, el análisis del uso de las prestaciones de paternidad resulta más complicado. Partimos del supuesto de que, mientras que en el caso de las prestaciones de maternidad por parto, que suponen más del $95 \%$ del total, todas las madres formalmente empleadas están obligadas a tener como mínimo seis semanas de descanso (con una prestación mínima garantizada para todas las madres que no cumplen requisitos previos de cotización), los padres disponen de libertad de elección para hacer uso o no de los permisos de paternidad. Tratándose de permisos acompañados por un subsidio equivalente al $100 \%$ de la base reguladora durante los días de suspensión del contrato de trabajo o cese en la actividad, podemos dar por sentado que la gran mayoría de padres tendrán un fuerte estímulo para su disfrute. Sin embargo, existen diversas circunstancias que pueden resultar adversas para el uso de los permisos por parte de los trabajadores: (1) no cumplir los requisitos de cotización ${ }^{9}$; (2) aun cumpliendo dichos requisitos es posible que algunas situaciones laborales como trabajar por cuenta propia o tener un contrato temporal no sean muy favorables para el disfrute de los permisos y de las prestaciones; (3) por último, es posible que algunos trabajadores no deseen tomar el permiso. De ahí el interés por indagar hasta qué punto y en qué circunstancias o contextos, o por qué motivos, los padres que

${ }^{9}$ Estar afiliados y en alta o en situación asimilada en algún régimen del sistema de la Seguridad Social y estar al corriente en el pago de las cuotas, así como tener cubierto un período mínimo de cotización de 180 días dentro de los 7 años inmediatamente anteriores a la fecha del inicio de dicha suspensión o permiso o, alternativamente, 360 días a lo largo de su vida laboral con anterioridad a la mencionada fecha. 
tienen derecho al descanso por nacimiento de un hijo solicitan y gozan o no de la prestación (Romero-Balsas, 2012; Escot Mangas \& Fernández-Cornejo, 2012) ${ }^{10}$.

El examen somero de los datos relativos a 2008-2011 nos permite constatar que el número absoluto de prestaciones de paternidad disfrutadas registró un cierto descenso en el período considerado ya que pasó de 279.756 en 2008 a 269.715 en 2011. Sin embargo, sospechamos que esta disminución podría ser consecuencia de la baja de la natalidad, del empleo o de ambos ${ }^{11}$. Con el fin de poder observar mejor la evolución de la tendencia en el uso de los permisos de paternidad proponemos elaborar una tasa de percepción de las prestaciones que descuente las variaciones en dichos fenómenos. Si aplicamos el porcentaje de la tasa de empleo de los varones de 25-54 años al número de nacimientos del año obtenemos el número padres potencialmente elegibles que constituyen la población de referencia para calcular la tasa de uso de los permisos. Así, por ejemplo en 2011 nacieron 471.999 niños en España. Con una tasa de empleo del 74,45\% para el grupo de edad 25-54 años tan sólo tres cuartas partes de los nacimientos serían elegibles (351.411). Ésta sería pues la población de referencia que se usaría para el cálculo de la tasa de uso cuyo valor resultante sería del $76,75 \%$. Este sencillo método también se puede usar para el cálculo de las tasas de uso de las prestaciones de maternidad percibidas por el padre. Uno de los inconvenientes de este método de estimación es que no tiene en cuenta el impacto de la paternidad sobre las tasas de empleo comentado anteriormente. Aunque sería deseable tomar en consideración dicho factor, en la práctica resulta muy difícil de operativizar y por tanto se ha omitido su influencia.

A pesar de esta limitación, este indicador tiene la ventaja de tomar en consideración las fluctuaciones paralelas que se han producido durante la crisis, tanto en el ámbito de la natalidad como del empleo. Su cálculo nos permite estimar las tasas de uso de las prestaciones de paternidad, así como de las prestaciones de maternidad percibidas por el padre, durante el período 2008-2011, para todas las comunidades autónomas, dado que los datos necesarios para ello se encuentran fácilmente disponibles en la base de datos del INE.

${ }^{10}$ Estos dos interesantes trabajos nos llegaron poco antes de cerrar la edición de este artículo, por lo que no pudimos tener en cuenta sus planteamientos en el diseño de nuestra investigación, aunque sí hemos contrastado con ellos nuestros resultados.

${ }^{11}$ En el conjunto de España el número de nacimientos registrados en el año 2008 fue de 519.779. En el año 2011 su número de había reducido a 471.999, lo que representa un descenso de 47.780 casos. Por otra parte, las tasas de empleo de los varones de 25 a 54 años pasaron del $84,36 \%$ en 2008 al $74,45 \%$ en 2011 , lo que supone una caída de 9,91 puntos. 


\subsection{Evolución y distribución territorial de las tasas de uso de las prestaciones de paternidad y maternidad percibidas por el padre}

El cálculo de las tasas de uso de las prestaciones de paternidad en el período 2008-2011 revela que, a pesar de la crisis, se ha producido un crecimiento continuado de su uso desde el inicio hasta el final. Asimismo, en el caso de las prestaciones de maternidad percibidas por el padre se observa una evolución muy parecida. Mientras que las tasas de uso de las prestaciones de paternidad pasaron del $63,8 \%$ en 2008 hasta el 76,7\% en 2011, en el mismo período las tasas de uso de las prestaciones de maternidad percibidas por el padre registraron una evolución del $1,27 \%$ al $1,65 \%$ (Gráfico 2).

La primera constatación es que los valores de las tasas de los dos tipos de prestaciones pertenecen a distintos órdenes de magnitud, lo cual ilustra con claridad meridiana la necesidad de impulsar los permisos de paternidad (exclusivos del padre) en contraste con los de maternidad percibidos por el padre (que se comparten con la madre y cuyo uso por el padre resta tiempo de disfrute a la madre), si se quiere de verdad fomentar la implicación del padre en el cuidado de los niños. De hecho, en el período 2008 y 2011 el porcentaje de prestaciones de maternidad percibidas por el padre sobre las percibidas por la madre oscila entre el $1,6 \%$ y el $1,8 \%$. Lo mismo podemos decir de las grandes diferencias existentes en lo que respecta al gasto en prestaciones de maternidad y de paternidad. En el período considerado los recursos públicos dedicados a prestaciones de paternidad con respecto al total (de paternidad y maternidad) representan entre un $10 \%$ y un $11 \%$. Haciendo una somera estimación de su coste para la economía del país obtendríamos tan sólo un $0,02 \%$ con respecto al PIB.

Por otra parte, el paralelismo de las líneas del gráfico es realmente sorprendente, máxime cuando se trata de variables con distintas escalas. Ello sugiere que la tendencia de ambos fenómenos podría venir impulsada por factores parecidos aunque operan con distintas intensidades en cada caso. 


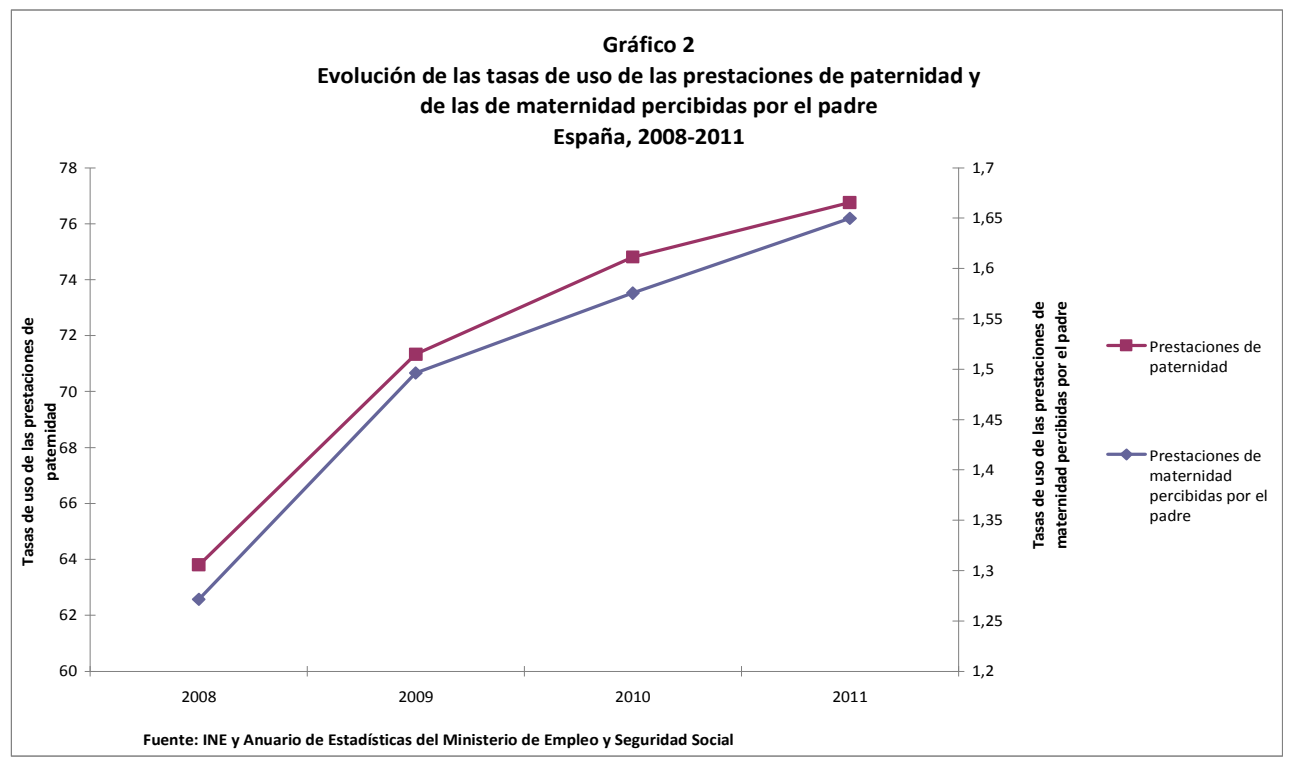

Las variaciones en las tasas de uso de las prestaciones de paternidad por comunidades autónomas son significativas. Con datos de 2011 mientras que en el País Vasco, Navarra, Aragón, la Rioja, Cantabria, Asturias y Cataluña las tasas de uso superan el $80 \%$, en Murcia, Baleares, Andalucía, Extremadura, Castilla-La Mancha y Comunidad Valenciana se hallan bajo la media. En lo que respecta a las tasas de uso de las prestaciones de maternidad percibidas por el padre, encontramos también notables variaciones según comunidades autónomas. Una vez más las tasas más altas aparecen en el País Vasco $(4,53 \%)$, Navarra (3,54\%), Cantabria y Asturias $(2,50 \%)$ frente a tasas inferiores a un $1 \%$ en Murcia $(0,56 \%)$ y Extremadura $(0,85 \%)$. 


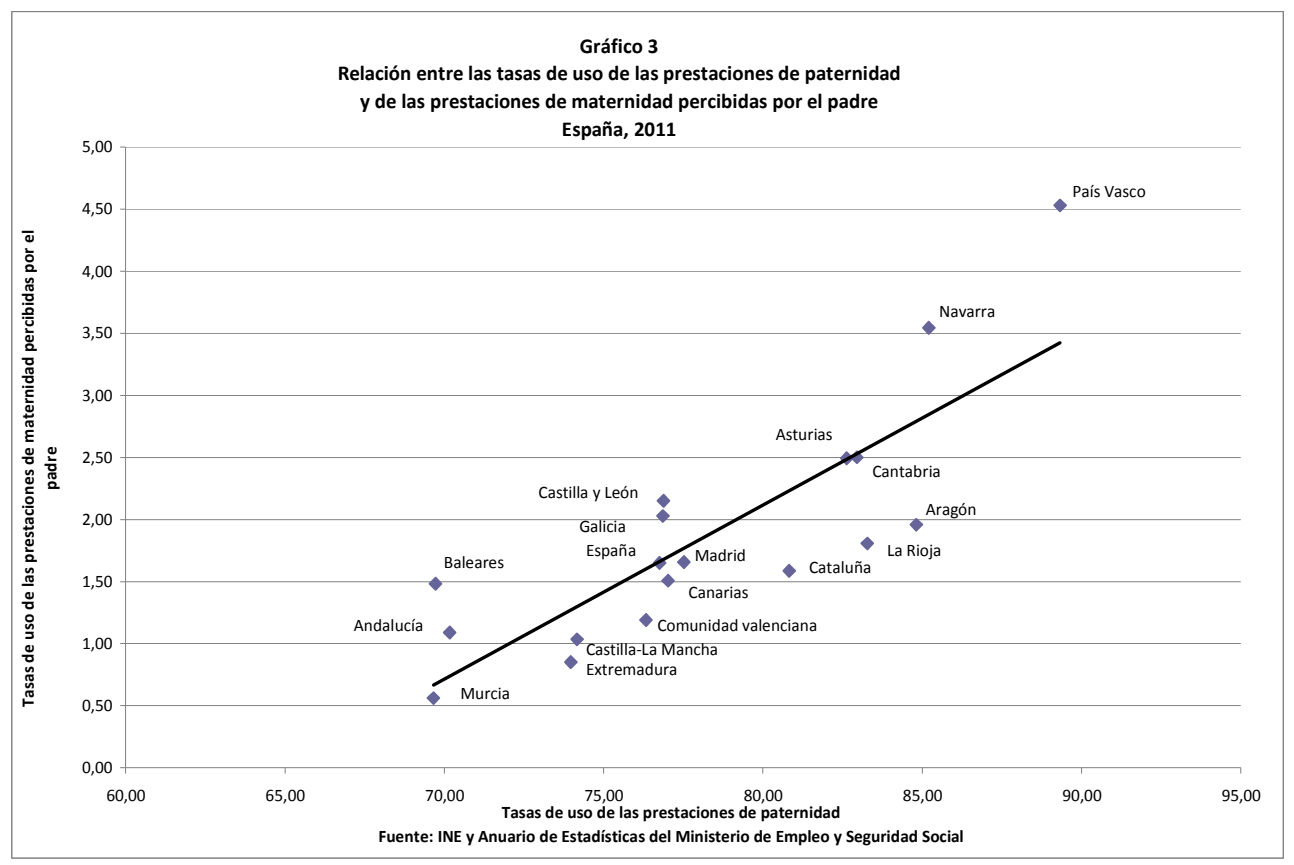

Según puede apreciarse en el Gráfico 3, los valores de las comunidades autónomas se alinean claramente a lo largo de la línea de tendencia, lo que resulta indicativo de la existencia de una elevada correlación positiva entre las tasas de uso de las prestaciones de paternidad y de las de maternidad percibidas por el padre. Esta relación es estable a lo largo del período estudiado con unos coeficientes que oscilan entre $\mathrm{R}=0,80$ y 0,90 , lo cual sugiere que los factores que subyacen a las tendencias de ambas variables son similares.

\subsection{Análisis de correlaciones sobre posibles factores que inciden en el uso de los permisos}

Con el fin de explorar algunos de esos posibles factores estructurales que pueden estar relacionados con la percepción de las prestaciones y el uso de las licencias correspondientes se ha hecho un análisis de correlaciones entre las tasas de uso de los permisos, por una parte, y ciertas variables que podrían ser potencialmente determinantes, por otra, utilizando las comunidades autónomas como unidades de observación. En el análisis se han descartado los valores de Ceuta y Melilla, ya que a menudo eran anómalos. Por supuesto, se trata de un análisis exploratorio ya que al trabajar con datos agregados y mediante un análisis de correlaciones no podemos establecer de manera concluyente ni el sentido de las posibles relaciones de causalidad ni los procesos individuales a través de los cuales se producen los efectos analizados. 
En la revisión de la literatura nos hemos centrado especialmente en posibles factores con datos anualmente disponibles para todas las comunidades autónomas en las bases de datos del INE o de otros organismos. En particular, hemos seleccionado dos grandes grupos de factores que podrían estar relacionados con el uso de los permisos por parte de los varones: (1) indicadores sobre sus niveles de instrucción y (2) indicadores sobre la calidad de su empleo.

Gracias a investigaciones americanas pioneras en el campo de la implicación paterna sabemos que la inclinación de los padres a cuidar de sus hijos pequeños es mayor entre los que tienen estudios universitarios que entre los pertenecientes a otros sectores de la población (McLanahan, 2004; Bianchi, 2000). En este sentido, podrían estar también más predispuestos a tomar permisos que los padres de niveles educativos inferiores. Ello puede darse por distintas razones: porque hayan tomado conciencia de su importancia al haber desarrollado una sensibilidad superior en relación con el cuidado de los niños, porque tengan actitudes de género más igualitarias o porque su nivel de formación les puede brindar mejores condiciones de trabajo (Escot Mangas y Fernández Cornejo eds, 2012). En sentido contrario, los padres con niveles de instrucción relativamente bajos o que hayan abandonado prematuramente sus estudios tendrán menores probabilidades de hacer uso de los permisos, máxime cuando es posible que su situación de precariedad en el empleo no les permita aprovecharse de ellos.

En este sentido, otros autores han destacado la posible relación entre el uso de los permisos y la calidad del empleo (Lapuerta, Baizán \& González, 2011; Escot Mangas y Fernández Cornejo, 2012; Romero-Balsas, 2012). Estar empleado en el sector público o en el privado, tener un contrato indefinido o temporal, ser asalariado o trabajar por cuenta propia y trabajar a tiempo completo o parcial pueden influir en la propensión a tomar permisos. 


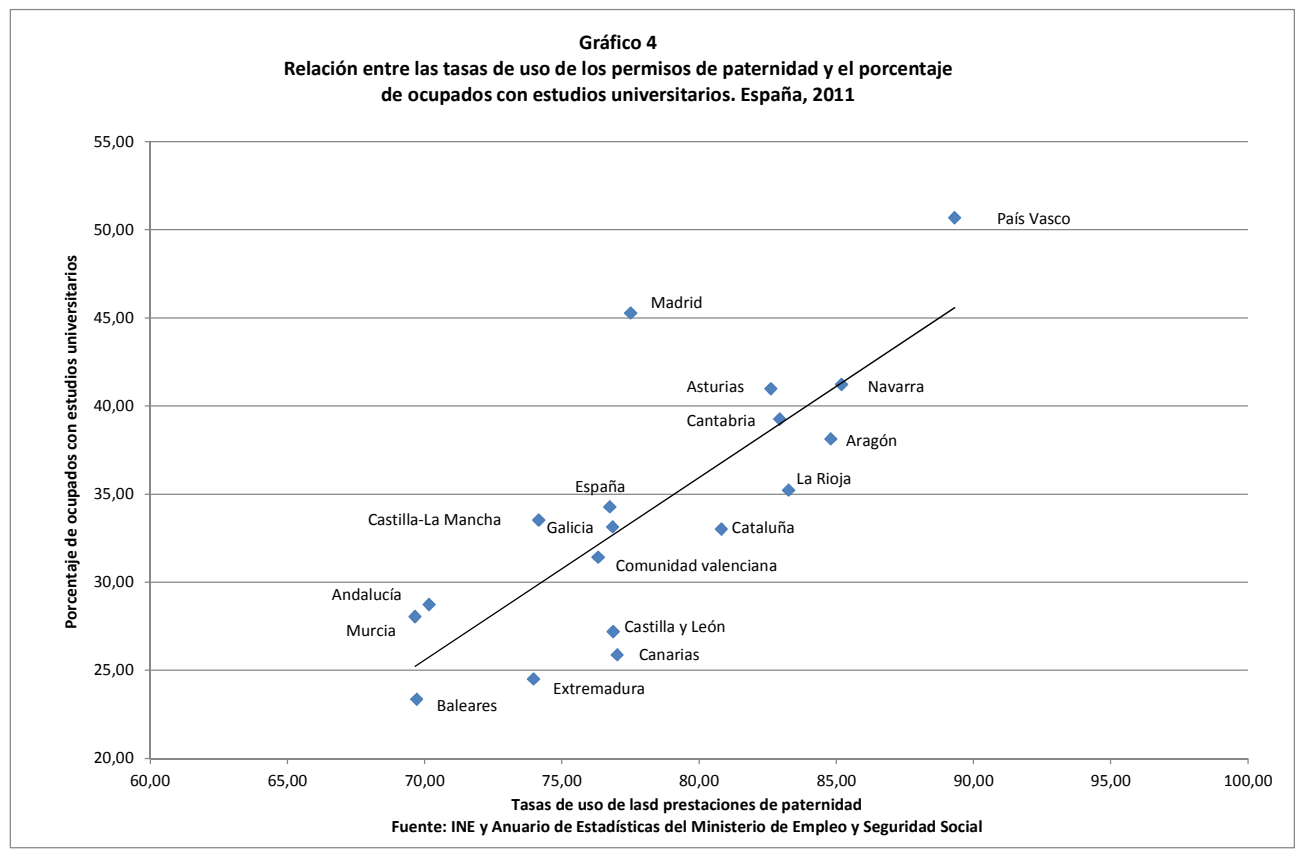

En relación con la primera hipótesis propuesta, las comunidades autónomas con una mayor proporción de hombres con elevados niveles educativos debieran presentar también mayores tasas de uso de los permisos. Tal como se puede observar en el Gráfico 4, se da una clara asociación positiva entre la tasa de uso de los permisos de paternidad y el porcentaje de ocupados con estudios universitarios. Además, el Gráfico 6 (más abajo) corrobora que esta fuerte asociación positiva se mantiene a lo largo del período estudiado e incluso se incrementa ligeramente con un coeficiente de correlación que pasa de $\mathrm{R}=0,75$ al $\mathrm{R}=0,80$. Esas mismas constataciones, aunque con una intensidad algo menor, valen también para el uso de los permisos de maternidad percibidos por el padre.

Sin embargo, no todos los estudios recientes hallan una relación clara y significativa entre el uso de los permisos y el nivel educativo de los padres. Según Escot Mangas y Fernández Cornejo eds (2012), si bien la variable 'nivel educativo alto' tiene un efecto positivo sobre la probabilidad de que el varón encuestado esté de permiso en la semana de referencia, según la EPA, el resultado obtenido no es estadísticamente significativo. Por otra parte, no existe unanimidad entre los trabajos de investigación sobre la relación entre altos niveles educativos y uso de los permisos. Aunque Romero-Balsas (2012) constata que los padres con estudios universitarios tomaron el permiso de paternidad con mayor frecuencia que el resto, su análisis de regresión logística arroja resultados contradictorios, en los que esta relación no aparece como significativa en la submuestra analizada de la "Encuesta sobre el uso de los permisos parentales en España, 2012". Habrá, pues, que seguir investigando para poder establecer, a través de nuevos análisis y en particular del 
análisis comparativo internacional, cuáles son las mediaciones existentes entre nivel educativo y uso de los distintos tipos de licencias parentales.

Para terminar este apartado sobre el uso de los permisos y los factores educativos, hemos examinado la relación existente entre las tasas de abandono educativo temprano de las comunidades autónomas y las tasas de uso de los permisos. En consonancia con los resultados anteriores, según se puede observar en el Gráfico 5, el análisis de correlaciones muestra una fuerte asociación negativa entre la percepción de prestaciones de paternidad y el abandono educativo temprano. Una vez más el País Vasco y Navarra aparecen en uno de los extremos mientras que Baleares, Murcia Andalucía y Extremadura se concentran en el otro. Por otra parte, en el caso de las prestaciones de maternidad percibidas por el padre, si bien los resultados son parecidos, en 2011 el coeficiente gana intensidad. A pesar de ello, en ambos casos, a lo largo del período considerado 2008-2011 se mantiene una fuerte correlación negativa entre los dos factores (ver más abajo Gráfico 6).

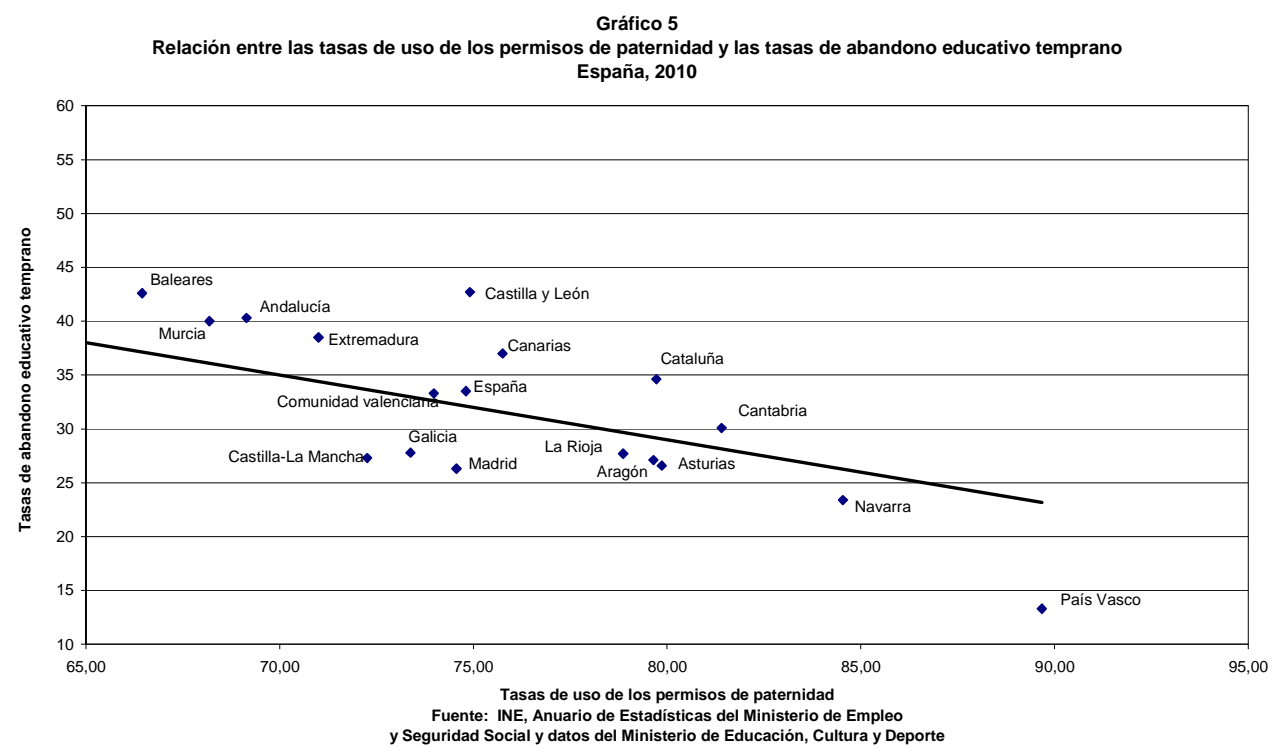

Teniendo en cuenta que el abandono educativo temprano constituye una de los factores determinantes de la precariedad en el empleo es muy posible que dicha asociación negativa tenga más que ver con dicho efecto que con los bajos niveles educativos. En consecuencia, para terminar este análisis de correlaciones hemos calculado los coeficientes de correlación entre las tasas de percepción de las prestaciones en las comunidades autónomas, por una parte, y las tasas de temporalidad, el porcentaje de trabajadores por cuenta propia y el porcentaje de trabajadores a tiempo parcial entre los ocupados, de otra. La inclusión del porcentaje de trabajadores a tiempo parcial como indicador de precariedad se debe al hecho de que, según datos de Eurostat, en España tres cuartas partes de los varones de mediana edad (25-59 años) que trabajan en esa modalidad de empleo lo 
hacen contra su voluntad. Esa proporción ha ido creciendo de forma espectacular desde el inicio de la crisis.

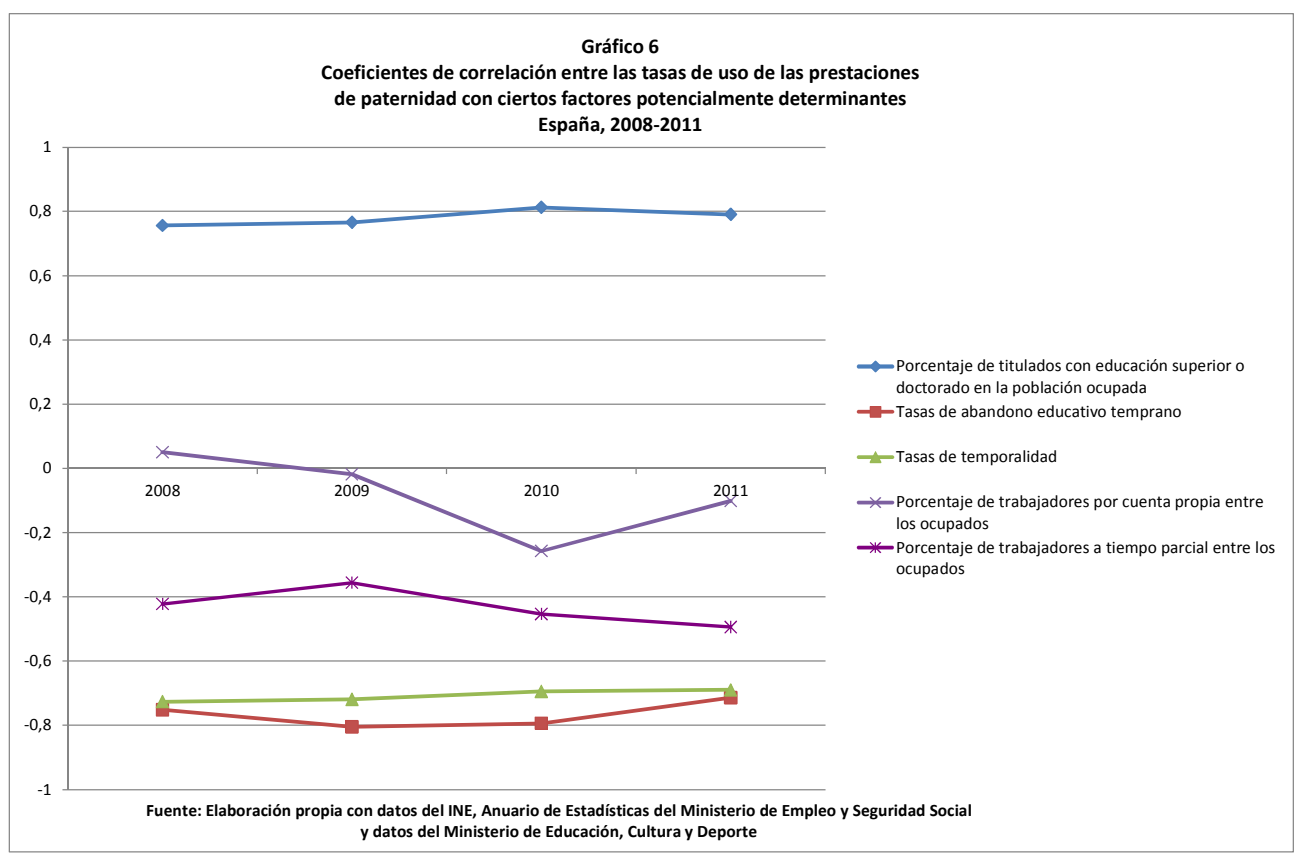

El Gráfico 6 ofrece los coeficientes de correlación entre las tasas de uso de los permisos de paternidad por Comunidades Autónomas y los factores sobre calidad del empleo entre 2008 y 2011. Entre dichos factores también aparecen los indicadores educativos anteriormente comentados. Según se puede observar, los factores con coeficientes de correlación relativamente más altos a lo largo de todo el período son las tasas de temporalidad y de abandono educativo temprano, por un lado, y la proporción de titulados con educación superior, por el otro. En cambio, el porcentaje de trabajadores por cuenta propia entre los ocupados exhibe una asociación nula en términos agregados, y el porcentaje de trabajadores a tiempo parcial presenta un coeficiente negativo relativamente bajo pero con tendencia ascendente. El análisis de correlaciones del uso de las prestaciones de maternidad percibidas por el padre por CCAA con dichos factores muestra resultados parecidos pero en el caso de las tasas de temporalidad la asociación negativa es de mucha menor intensidad.

Estos resultados concuerdan sólo parcialmente con los obtenidos por otros investigadores (Lapuerta, Baizán \& González, 2011; Escot Mangas y Fernández Cornejo eds, 2012; Romero-Balsas, 2012). Así, aunque todos insisten en la importancia de la estabilidad en el empleo para el uso de los permisos, el hecho de ser trabajador autónomo resulta ser un claro obstáculo para ello, (según los resultados obtenidos por Romero-Balsas, 2012), lo cual contrasta con nuestros 
resultados de que a nivel agregado la proporción de autónomos no parece tener el mismo peso explicativo. Por último, nuestros análisis sugieren la conveniencia de incluir el trabajo a tiempo parcial como un factor negativo para el uso de los permisos en la medida en que su coeficiente está ganando intensidad en los últimos años, particularmente entre los varones y de forma no voluntaria.

\section{Conclusiones}

En la mayoría de países, paternidad y maternidad tienen un impacto diverso y a menudo de signo opuesto sobre las trayectorias laborales. En los países con políticas de conciliación más activas e igualitarias se está consiguiendo una relación positiva entre empleo y parentalidad tanto para hombres como para las mujeres. Con datos anteriores a la crisis, hemos mostrado como en España el nacimiento de un primogénito todavía marca un punto de inflexión a partir del cual divergen en gran medida el conjunto de trayectorias laborales femeninas y masculinas en nuestro país. Mientras que el acceso a la paternidad se corresponde con una etapa vital de desarrollo profesional para la mayoría de hombres con hijos pequeños (en torno a dos tercios), éste se observa en una menor proporción de madres (44\%) y se relaciona la maternidad con una precarización de las trayectorias laborales de un tercio de las madres empleadas.

En la actual situación de crisis, la evolución de las tasas de empleo apuntan a un mayor esfuerzo de las madres por mantenerse ocupadas, y a una mayor precariedad de las condiciones laborales de los hombres, apuntando ambas tendencias hacia nuevas combinatorias y contextos de articulación entre cuidado de los hijos y empleo en los hogares españoles, más igualitarios entre hombres y mujeres, pero también más precarios y desiguales entre grupos sociales.

En este contexto, las políticas de conciliación de la vida laboral y familiar son importantes tanto para los hombres como para las mujeres y, según qué medidas pueden actuar como mecanismos de flexi-seguridad para todos o bien acentuar las desigualdades entre trabajadores con mejores condiciones de trabajo y aquellos afectados por una mayor precariedad. Las licencias de maternidad y de paternidad responden al primer tipo, mientras que las excedencias por cuidado de hijos, aunque estén incentivadas fiscalmente o reciban en algunas comunidades autónomas ciertas prestaciones menores responden al segundo tipo, como han mostrado distintos resultados de la investigación social, ya que refuerzan la especialización de funciones de género y sólo facilitan la conciliación de la vida laboral y familiar de los trabajadores con una posición estable en el mercado de trabajo.

En este artículo hemos desarrollado una metodología para poder estimar el uso relativo de los permisos de paternidad. El indicador que hemos elaborado muestra que, al contrario de lo que cabría esperar, la crisis económica no ha tenido impacto en la percepción de las prestaciones de paternidad. Así, en el período 2008-2011, a pesar de la disminución de la natalidad y del empleo, nuestro cálculo de las tasas de uso de las prestaciones de paternidad sobre la población masculina empleada revela 
que se ha producido un crecimiento continuado de su uso. Mientras que las tasas de percepción de las prestaciones de paternidad pasaron del 63,8\% en 2008 hasta el $76,7 \%$ en 2011 , en el mismo período las tasas de las prestaciones de maternidad percibidas por el padre registraron una evolución del 1,27\% al 1,65\%. Los distintos órdenes de magnitud a que pertenecen los valores de las tasas de ambos tipos de prestaciones confirman el acierto de la introducción de la licencia por paternidad en España, en contraste con la posibilidad preexistente de transferencia al padre de una parte de la licencia por maternidad, ya que para que haya implicación y utilización de las licencias parentales por parte de los hombres, éstas han de ser permisos individuales y exclusivos, no derivados de los derechos y cotizaciones sociales de la madre.

En España a partir de 2007 las políticas de licencias parentales empiezan a ser percibidas como un medio para promover la implicación paterna en las tareas de cuidado y crianza de los niños. Sin embargo, más allá de las políticas incentivadoras del uso paterno de las licencias laborales, que se han convertido en emblemáticas en toda Europa, el análisis de correlaciones realizado, orientado a la identificación y seguimiento de factores socioestructurales que impulsan su uso, a partir de la base de los datos publicados anualmente de prestaciones de paternidad y maternidad, muestra que las comunidades autónomas en las que existe una mayor participación de los padres se corresponden no sólo con aquellas con políticas de igualdad de género más activas, sino también con unos sistemas productivos y laborales de mayor calidad en términos de niveles educativos de la población activa y condiciones laborales.

El análisis de la posición relativa de nuestro país en el conjunto de la Unión Europea, sugiere pistas sobre aquellas tendencias que pueden ser consideradas como puntos fuertes u oportunidades. En España se está generalizando el empleo materno con menores diferenciales de impacto de la maternidad que en otros países de la UE -salvo en los niveles educativos más bajos- $y$, por lo tanto, vale la pena que, siendo éste el punto de partida, se desarrollen políticas sociales que apoyen la conciliación del empleo con la maternidad y la paternidad y las tareas de cuidado que comportan, en una perspectiva de igualdad de género tanto dentro como fuera del hogar.

Los sistemas de licencias parentales retribuidos y limitados en el tiempo, que se instrumentan dando opción a su uso a tiempo completo o parcial, pero manteniendo plenamente el estatus de empleo y retribución, impulsan un reparto más igualitario de las responsabilidades de cuidado entre hombres y mujeres y han mostrado ser los sistemas más eficientes en términos de impacto positivo sobre el empleo facilitando su uso por parte de hombres y mujeres, sin menoscabo de la consolidación de las carreras profesionales y de cotización que desarrollan padres y madres tras el nacimiento de sus hijos. 


\section{Bibliografía}

Alberdi, I. y P. Escario (2007). Los hombres jóvenes y la paternidad. Bilbao: Fundación BBVA.

Bianchi, S. M. (2000). Maternal Employment and Time with Children: Dramatic Change or Surprising Continuity?. Demography 37 (4): 401-414.

Björnberg, U. \& A.-K. Kollind (eds.) (1996). Men's Family Relations. Stockholm: Almqvist \& Wiksell International.

Brandth, B. \& E. Kvande (2009). Gendered or Gender-Neutral Care Politics for Fathers? The Annals of the American Academy of Political and Social Science, Vol. 624, Núm. 1, 177-189.

Cabrera, N. \& H. E. Peters (2000). Public Policies and Father Involvement. Marriage \& Family Review, Vol. 29, Núm. 4, 295-314.

Castro, C. y M. Pazos (2008). Permiso de maternidad, de paternidad y parentales en Europa: Algunos elementos para el análisis de la situación actual. En M. Pazos (eds.) Economía e igualdad de género: Retos de la Hacienda Pública en el siglo XXI. (pp. 185-222). Madrid: Instituto de Estudios Fiscales.

Daly, M. (2011). 'What Adult Worker Model? A Critical Look at Recent Social Policy Reform in Europe from a Gender and Family Perspective. Social Politics. Vol. 19, Núm. 1, 1-23.

Deven, F. \& P. Moss (eds) (1999). Parental Leave: Progress or Pitfall? Policy and Research Issues in Europe. The Hague/Brussels: NIDI/CBGS Publications.

Duvander, A.-Z. \& A.-C. Jans (2008). Consequences of Fathers Parental Leave Use: Evidence from Sweden. Stockholm Research Reports in Demography 2008: 9. Stockholm: Stockholm University.

Escobedo, A. \& Meil, G. (2013). Spain country note, in: P. Moss (ed.) International Review of Leave Policies and Research 2013. Available at: http://www.leavenetwork.org/lp_and_r_reports/

Escobedo, A. (2008). Spain en: Moss P. \& Korintus, M. (eds.) International Review of Leave Policies and Related Research 2008 Employment Relations Research Series. Department for Business, Enterprise \& Regulatory Reform, UK.

Escobedo, A. (2009). Leave policies and public systems of care for children under three years old and their families in the European Union. Tesis doctoral amb mención europea. Departamento de Sociologia. Universidad Autónoma de Barcelona. 
Escobedo, A. (2013). Conciliating Parents' Labor and Family Life. In A. Ben-Arieh, F. Casas, I. Frønes and J. E. Korbin (eds) Handbook of Child Well-Being. Theories, Methods and Policies in Global Perspective. (pp. 2153-2172). Vol. 4. Dordrecht: Springer.

Escobedo, A. y L. Navarro (2007). Una evaluación del sistema de licencias parentales y por motivos familiares en España desde la perspectiva de género. Comunicación presentada al Grupo de Trabajo de Sociología del Género del Congreso de la Federación Española de Sociología. Barcelona, 15 de Septiembre de 2007.

Escobedo, A., L. Flaquer \& L. Navarro (2012). The social politics of fatherhood in Spain and France: A comparative analysis of parental leave and shared residence. Ethnologie Française, Vol. 42, Núm. 1, 117-126.

Escobedo, A., L. Navarro y L. Flaquer (2007). Perspectivas de desarrollo y evaluación de las políticas de licencias parentales y por motivos familiares en España y en la Unión Europea. Madrid: FIPROS.

Escobedo, A., L. Navarro y L. Flaquer (2008). El impacto de la maternidad y la paternidad en el empleo y uso de prestaciones de la Seguridad Social en España: Itinerarios laborales de madres y padres en hogares con menores de tres años. Análisis de cambios y discontinuidades a partir de la Muestra Continua de Vidas Laborales 2005. Madrid: FIPROS.

Escot Mangas, L., y Fernández Cornejo, J. A. (eds.) (2012) Una evaluación de la introducción del permiso de paternidad de 13 días. ¿Ha fomentado una mayor corresponsabilidad en el ámbito del cuidado de los hijos pequeños? Madrid: Colección Estudios e Investigaciones, Instituto de la Mujer. http://www.ucm.es/centros/cont/descargas/documento38578.pdf

European Commission (2010). Indicators for monitoring the Employment Guidelines including indicators for additional employment analysis. Compendium 2010. Brussels: DG Employment, Social Affairs and Equal Opportunities.

Flaquer, L. (2013). Family-related factors influencing child well-being. In A. BenArieh, F. Casas, I. Frønes, and J. E. Korbin (eds.) Handbook of Child Well-being. Theories, Methods and Policies in Global Perspective. (pp. 2229-2255). Vol. Dordrecht: Springer.

Fusulier, B. \& J. Marquet (2007). Présentation: Hommes, pères et travailleurs. Recherches sociologiques et anthropologiques 2: 1-7.

Haataja, A. (2009). Fathers use of paternity and parental leave in the Nordic countries. Helsinki: The Social Insurance Institution of Finland Research Department. Online working papers 2/2009. 
Hobson, B. (ed) (2002). Making Men into Fathers: Men, Masculinities and the Social Politics of Fatherhood. Cambridge: Cambridge University Press.

Kamerman, S. B. \& P. Moss (eds) (2009). The politics of parental leave policies: Children, parenting, gender and the labour market. Bristol: The Policy Press.

Lammi-Taskula, J. (2006). Nordic men on parental leave: Can the welfare state change gender relations? In Ellingsaeter, A. L. A. and Leira (eds) Politicising Parenthood in Scandinavia: Gender Relations in Welfare States. (pp.79-100. Bristol: Policy Press..

Lapuerta, I., P. Baizán \& M. J. González (2011). Individual and institutional constraints: An analysis of parental leave use and duration in Spain. Population Research and Policy Review, Vol. 30, Núm. 2, 185-210.

Leira, A. (1992). Welfare States and Working Mothers: The Scandinavian Experience. Cambridge: Cambridge University Press.

Leira, A. (1998). Caring as Social Right: Cash for Child Care and Daddy Leave. Social Politics, Vol. 5, Núm. 3, 362-377.

Lewis, J. (1992). Gender and the Development of Welfare Regimes. Journal of European Social Policy, Vol. 2, Núm. 3, 159-173.

Lewis, J. (2006). Introduction: Children in the Context of Changing Families and Welfare States. In J. Lewis (ed.) Children, Changing Families and Welfare States. (pp. 1-24). Cheltenham: Edward Elgar.

Lewis, J. (ed.) (1997). Lone Mothers in European Welfare Regimes: Shifting Policy Logics. London and Philadelphia: Jessica Kingsley Publishers.

Lewis, J., M. Campbell \& C. Huerta (2008). Patterns of paid and unpaid work in Western Europe: Gender, commodification, preferences and the implications for policy. Journal of European Social Policy, Vol. 18, Núm. 1, 21-37.

Marsiglio, W. \& K. Roy (2012). Nurturing Dads: Social Initiatives for Contemporary Fatherhood. New York: Russel Sage Foundation.

Martial, A. (2012). Paternités contemporaines et nouvelles trajectoires familiales. Ethnologie française, Vol. 42, Núm. 1, 105-116.

McLanahan, A, L. Tach \& D. Schneider (2013). The Causal Effects of Father Absence. Annual Review of Sociology, Vol. 39, 399-427.

McLanahan, S. (2004). Diverging Destinies: How Children Are Faring Under the Second Demographic Transition. Demography, Vol. 41, Núm. 4, 607-627. 
Meil, G. (1997). La participación masculina en el cuidado de los hijos en la nueva familia urbana española. Papers. Revista de Sociología, Vol. 53, 77-99.

Meil, G. (2011). El uso de los permisos parentales por los hombres y su implicación en el cuidado de los niños en Europa. Revista Latina de Sociología, Vol. 1, 61-97.

Meil. G. (2013). La utilización por los hombres de los permisos parentales y su implicación en el cuidado de niños. Contribución al XI Congreso Español de Sociología.

Moss, P. (2008). Making parental leave parental: An overview of policies to increase fathers'use of leave. In P. Moss and M. Korintus (eds) International Review of Leave Policies and Related Research 2008. London: Department for Business, Enterprise and Regulatory Reform. Employment Relations Research Series No. 100.

Moss, P. (2013). 2013 Annual Review of Leave Policies and Research. Descargado el 18/7/2013 en http://www.leavenetwork.org/lp_and_r_reports/

O’Brien, M. (2004). 'Social Science and Public Policy Perspectives on Fatherhood in the European Union'. In M. E. Lamb (ed.) The Role of the Father in Child Development. (pp. 121-145). Hoboken, N.J.: John Wiley \& Sons.

O'Brien, M. (2009). Fathers, Parental Leave Policies and Infant Quality of Life: International Perspectives and Policy Impact. The Annals of the American Academy of Political and Social Science, Vol. 624, Núm. 1, 190-213.

O'Brien, M. (2012). Work-Family Balance Policies. Background paper for the preparation of the twentieth anniversary of the International Year of the Family in 2014. UNDESA, New http://social.un.org/index/LinkClick.aspx?fileticket=cwncEgAZz9A\%3d\&tabid=1733.

O’Brien, M., B. Brandth \& E. Kvande (2007). Fathers, work and family life: Global perspectives and new insights. Community, Work and Family 10: 375-386.

OCDE (2011) Doing better for families, http://www.oecd.org/social/soc/doingbetterforfamilies.htm

Orloff, A. S. (1993). Gender and the social rights of citizenship: The comparative analysis of gender relations and welfare states. American Sociological Review, Vol. 58, Núm. 3, 303-328.

Orloff, A. S. (1996). Gender and the welfare state. Annual Review of Sociology, Vol. 22, 51-78. 
Orloff, A. S. (2009). Gendering the comparative analysis of welfare states: An unfinished agenda. Sociological Theory, Vol. 27, Núm. 3, 317-343.

Pfau-Effinger, B (2004). Development of Culture, Welfare States and Women's Employment in Europe. Aldershot: Ashgate.

Pfau-Effinger, B. (2005). Welfare State Policies and the Development of Care Arrangements. European Societies, Vol. 7, Núm. 2, 321-347.

Romero-Balsas, P. (2012). Fathers Taking Paternity Leave in Spain: Which Characteristics Foster and Which Hampers the Use of Paternity Leave. Sociologia e Politiche Sociali, Vol. 15, 106-131.

Rostgaard, T. (2002). Setting time aside for the father: Father's leave in Scandinavia. Community, Work and Family, Vol. 5, Núm. 3, 343-364.

Salazar, O. (2013). Masculinidades y ciudadanía: Los hombres también tenemos género. Madrid: Dykinson.

Thévenon, O. (2011) Family Policies in OECD Countries: A Comparative Analysis, Population and Development Review, Vol. 37, Núm. 1, 57-87.

Truc, G. (2006). La paternité en maternité: Une étude par observation. Ethnologie Française, Vol. 36, Núm. 2, 341-349.

Valiente, C. (1997). ¿Algo más que "ganadores del pan"?: El papel de los hombres en el ámbito familiar en España (1975-1996)'. Revista Española de Investigaciones Sociológicas, Vol. 79, Núm. 1, 221-243.

Valiente, C. (2005). The Changing Roles of Men in Families in Spain. In M. Threlfall, C. Cousins and C. Valiente. Gendering Spanish Democracy. (pp. 187-203). London and New York: Routledge.

Wall, K \& A. Escobedo (2013). Parental leave policies, gender equity and family well-being in Europe: A comparative perspective. In A. Moreno Mínguez (ed.) Family Well-Being: European Perspectives. (pp. 103-129). Dordrecht: Springer,

Wall, K. \& Escobedo, A. (2009) Portugal and Spain: two pathways in Southern Europe, en: Kamerman, S. \& Moss, P. (eds.) The politics of parental leave policies: Children, parenting, gender and the labour market. (pp. 207-226.) Bristol. The Policy Press.

Wilson, K. R. \& M. R. Prior (2011). Father involvement and child well-being. Journal of Paediatrics and Child Health, Vol. 47, 405-407. 